\title{
Regional myocardial motion in patients with mild cognitive impairment: a pilot study
}

Heng Ma ${ }^{1,2+}$, Jun Yang ${ }^{1+}$, Haizhu Xie ${ }^{1}$, Jing Liu' ${ }^{1}$, Fang Wang ${ }^{1}$, Xiao Xu' ${ }^{1}$, Wei Bai ${ }^{1}$ and Kai Lin ${ }^{2^{*}}$ (D)

\begin{abstract}
Background: Cardiovascular disease (CVD) is a risk factor for cognitive impairment in the elderly. Manifestations of subclinical CVDs can be found in patients with cognitive impairment. The aim of the present study was to test the hypothesis that patients with mild cognitive impairment (MCI) have different magnetic resonance imaging (MRI)-derived regional myocardial motion indices compared with healthy controls.
\end{abstract}

Methods: Eleven MCl patients (age, 65.5 years \pm 5.9 ; range, 55-81 years old) and 11 sex-/age-matched healthy volunteers were enrolled. All of the participants underwent a head MRI and cardiac MRI. Global cortical atrophy (GCA) was graded on the head MRI. The left ventricular ejection fraction (LVEF) and regional strain, strain rate, displacement and velocity were measured on cine images. The GCA scores, global cardiac function and regional myocardial motion indices were compared between $\mathrm{MCl}$ patients and healthy controls using the t-test.

Results: $\mathrm{MCl}$ patients had a higher GCA score than healthy controls $(p=0.048)$. However, there was no significant difference in LVEF between $\mathrm{MCl}$ patients and controls. Compared to healthy controls, $\mathrm{MCl}$ patients had a lower peak radial strain $(29.1 \% \pm 24.1 \%$ vs. $46.4 \% \pm 43.4 \%, p<0.001)$, lower peak diastolic radial strain rate $\left(3.2 \pm 2.4 \mathrm{~s}^{-1} \mathrm{vs} .6 .0 \pm 3.0 \mathrm{~s}^{-1}\right.$, $p<0.001)$, lower peak diastolic circumferential strain rate $\left(2.5 \pm 2.1 \mathrm{~s}^{-1}\right.$ vs. $\left.3.2 \pm 2.1 \mathrm{~s}^{-1}, p=0.002\right)$, lower peak systolic radial displacement ( $4.2 \pm 2.2 \mathrm{~mm}$ vs. $5.2 \pm 3.3 \mathrm{~mm}, p=0.002$ ), lower peak diastolic radial velocity $(31 \pm 18 \mathrm{~mm} / \mathrm{s} \mathrm{vs.} 45 \pm 33 \mathrm{~mm} / \mathrm{s}$, $p<0.001)$, and lower peak diastolic circumferential velocity ( $178 \pm 124$ degree/s vs. $217 \pm 131$ degree/s, $p=0.005$ ).

Conclusion: MRI-derived regional myocardial strain, strain rate and velocity were found to be different between MCI patients and healthy controls. Regional myocardial motion indices have the potential to become novel quantitative imaging biomarkers for representing the risk of neurodegenerative disorders, such as Alzheimer's disease (AD).

Keywords: Mild cognitive impairment, Cardiac MRl, Regional myocardial motion

\section{Background}

Alzheimer's disease (AD) is the most common cause of cognitive impairments, including mild cognitive impairment (MCI) and dementia, in older adults [1]. Cardiovascular disease (CVD) has been considered a prominent contributor to $\mathrm{AD}$ in the elderly [2,3]. An intuitive mechanistic link between CVD and AD is chronic brain hypoxia due to progressive heart dysfunction [4]. Nonetheless, the causal relationship between CVD and AD is still not fully understood and there is no consensus on whether treating CVDs can reduce AD risk. "Health United States Report 2016" (www.cdc.gov/nchs/data/hus/hus16.pdf) showed

\footnotetext{
* Correspondence: kai-lin@northwestern.edu

${ }^{\dagger}$ Equal contributors

2Department of Radiology, Northwestern University Feinberg School of

Medicine, 737 N Michigan Ave, Ste 1600, Chicago, IL 60611, USA

Full list of author information is available at the end of the article
}

that, from 2000 to 2015 , deaths per 100,000 people (after age adjustment) from CVDs decreased substantially (from 257.6 to 168.5 ) while deaths from AD increased dramatically (from 18.1 to 29.4). These outcomes suggest that advancements in cardiovascular treatment strategies could successfully extend the lives of CVD patients but did not directly improve AD outcomes. By the time CVD is detected and treated, its impact on the brain might have already been irreversible, suggesting that early intervention for subclinical CVD is critical to prevent $A D$ [1]. Unfortunately, conventional quantitative cardiovascular indices of global cardiac function, such as left ventricular ejection fraction (LVEF), are insufficient to link subclinical CVD to AD [5, 6]. A logical explanation is that myocardial ischemia and dysfunction in the early stage of CVD can occur at a regional level and might not initially impact the global cardiac

(c) The Author(s). 2018 Open Access This article is distributed under the terms of the Creative Commons Attribution 4.0 International License (http://creativecommons.org/licenses/by/4.0/), which permits unrestricted use, distribution, and 
function. Therefore, cardiac imaging biomarkers that can better bridge subclinical CVD to AD are highly desired.

Recently, quantitative biomechanical indices acquired using magnetic resonance imaging (MRI), such as strain, strain rate, displacement and velocity, have been adopted to comprehensively describe abnormal myocardial motion patterns [7]. Compared with global indices, regional myocardial motion indices seem to be more appropriate candidates for describing subtle cardiac changes affiliated with subclinical CVDs. However, whether regional myocardial motion indices have the potential to indicate the risk of $\mathrm{AD}$-related cognitive impairments has not been fully demonstrated. To address this unmet clinical need, we investigated regional myocardial motion indices in MCI patients who do not have documented CVDs using quantitative cardiac MRI. The aim of the present study was to test the hypothesis that MCI patients have different regional myocardial motion indices compared with healthy controls.

\section{Methods \\ Participants}

This prospective study was approved by the local ethics review board and written informed consent was obtained from all of the participants. From 2012 to 2017, the medical records of MCI patients in our institution were reviewed. All of the MCI patients were clinically diagnosed at our institution by experienced neurologists according to the guideline published by National Institute on Aging-Alzheimer's Association workgroups in 2011 [8]. Specifically, the diagnosis of MCI was based on the following criteria: 1 ) reasonable concern from the patient or his/her relatives regarding a recent change in cognition/behavior compared with the patient's previous level; 2) the impairment of cognitive in at least one major domain, such as executive function, episodic memory, attention, visuospatial capability, or language skills. The impairment should be beyond the patient's educational background and age scale; and 3) the patients should preserve independence in daily abilities with minimal aid or assistance. There is no evidence of significant impairment in social or occupational functioning and he/she should not be demented. The inclusion criteria for participants were: 1 ) $\geq 55$ years old; 2) without documented structural CVDs; 3) without dementia or other psychiatric diseases; 4) without stroke or brain tumor; and 5) without other life threatening conditions that can significantly affect the cardiovascular system, including kidney dysfunction and rheumatologic diseases. The exclusion criteria were: 1) participants with contradictions of MRI. In total, 11 patients with MCI and 11 sex- and age-matched $( \pm 2$ years of difference allowed) healthy volunteers were enrolled in this study. The control group consisted of individuals without either history or signs of structural CVDs or cognitive impairments. See Table 1 for demographic information of the participants.

\section{MR protocols}

All of the participants were imaged with a $3.0 \mathrm{~T}$ clinical MRI scanner (Signa EXCITE, GE Healthcare, Waukesha, WI, USA) by two certified MRI technicians. The cardiac MRI and head MRI were consequently performed on the same day with a 4-element phased-array surface coil and a head coil, respectively. Blood pressure (BP), body weight and height were measured for each participant immediately before MRI scans. Related health information for assessing traditional cardiovascular risks was collected by checking the medical history.

\section{Cardiac MRI procedure}

After localization of the heart, cine images were acquired by using a Fast Imaging Employing Steady-state Acquisition (FIESTA) sequence at two-chamber, three-chamber and short-axis views. The short-axis cine images covered the heart from base to apex with 10-12 slices. The imaging parameter included: TR/TE $=3.6 / 1.6 \mathrm{~ms}$, field of view $(\mathrm{FOV})=380 \times 380 \mathrm{~mm}$, matrix $=512 \times 512$, slice thickness $=8 \mathrm{~mm}$. Twenty retrospective phases were constructed within a cardiac cycle.

\section{Head MRI procedure}

A three-plane localizer was used to prepare the imaging planes. A fast spin echo (FSE) sequence was run on axial and sagittal views to obtain $\mathrm{T} 1$ weighted images (T1WI) and T2 weighted images (T2WI) covering the whole brain. The imaging parameters included: T1WI: TR/TE $=750$ / $25 \mathrm{~ms}$, FOV $=200 \times 200 \mathrm{~mm}^{2}$, matrix $=512 \times 512$, slice thickness $=8 \mathrm{~mm}$. T2WI: TR/TE $=3500 / 100 \mathrm{~ms}$, FOV $=$ $200 \times 200 \mathrm{~mm}^{2}$, matrix $=512 \times 512$, slice thickness $=8 \mathrm{~mm}$.

\section{MR image analysis Head MRI analysis}

All of the images underwent regularly checks by reviewer \#1 (H.M, with 10-year experience in clinical radiology) before cardiac MRI analysis was started. Global cortical atrophy (GCA, four-point scale, score 0-3) scores were then graded semi-quantitatively by reviewer \#1 based on T1WI and T2WI images using criteria from the existing literature [9]. Grades of GCA were determined by judging the widening of the sulci and gyral volume loss by reviewer \#1. Normal sulci received a GCA grade of 0 , slight widening of sulci was considered to have a GCA of 1, gyral volume loss was assigned a GCA of 2 and prominent sulci widening with substantial volume loss was categorized as a GCA of 3 . 
Table 1 General Characteristics of $\mathrm{MCl}$ Patients and Controls*

\begin{tabular}{llll}
\hline & MCl patients $(n=11)$ & Healthy controls $(n=11)$ & $p$ values* \\
\hline Age (years) & $65.5 \pm 5.9$ & $66.4 \pm 8.3$ & NS \\
Male (\%) & $6(55)$ & $6(55)$ & NS \\
Hypertension (\%) & $2(18)$ & $2(18)$ & NS \\
Diabetes mellitus (\%) & $3(27)$ & $4(36)$ & NS \\
Smokers (\%) & $2(18)$ & $3(27)$ & NS \\
Systolic blood pressure $(\mathrm{mmHg})$ & $134.6 \pm 8.9$ & $126.3 \pm 15.6$ & $\mathrm{NS}$ \\
Diastolic blood pressure $(\mathrm{mmHg})$ & $80.3 \pm 7.6$ & $82.5 \pm 9.2$ & $\mathrm{NS}$ \\
Body mass index $(\mathrm{Kg} / \mathrm{m} 2)$ & 26.8 & 25.7 & NS \\
\hline
\end{tabular}

* "NS" indicates that no statistically significant difference was found between the two groups

\section{Cardiac MRI analysis}

All of the cardiac images were analyzed using Circle CVI42 software (Calgary, Canada) on a dedicated workstation (Dell, Precision T1650, USA). After short-axial and four-chamber cine images were loaded, reviewer \#1 manually traced the endocardial and epicardial borders of the left ventricle (LV) to define region of interest (ROI) at the end-systolic and end-diastolic phases, respectively. MRI-derived global cardiac function and regional myocardial motion indices, such as LVEF, strain, strain rate, displacement and velocity, were automatically calculated based on the ROIs. Regional motion indices in the radial and circumferential directions were mapped on an AHA 16-segment LV model. Long-axis motion indices were mapped on a 6-segment LV model. Measures for each participant were output as a single Microsoft Excel spreadsheet for further analysis.

\section{Quality control (QC) for measurements of regional myocardial motion indices}

Reviewer \#1 re-analyzed the data of all of the cases after a one-month interval to test the intra-observer agreement in measurements of regional myocardial motion indices. Reviewer \#2 (K.L, with 13-years of experience in clinical radiology) independently analyzed all of the cases

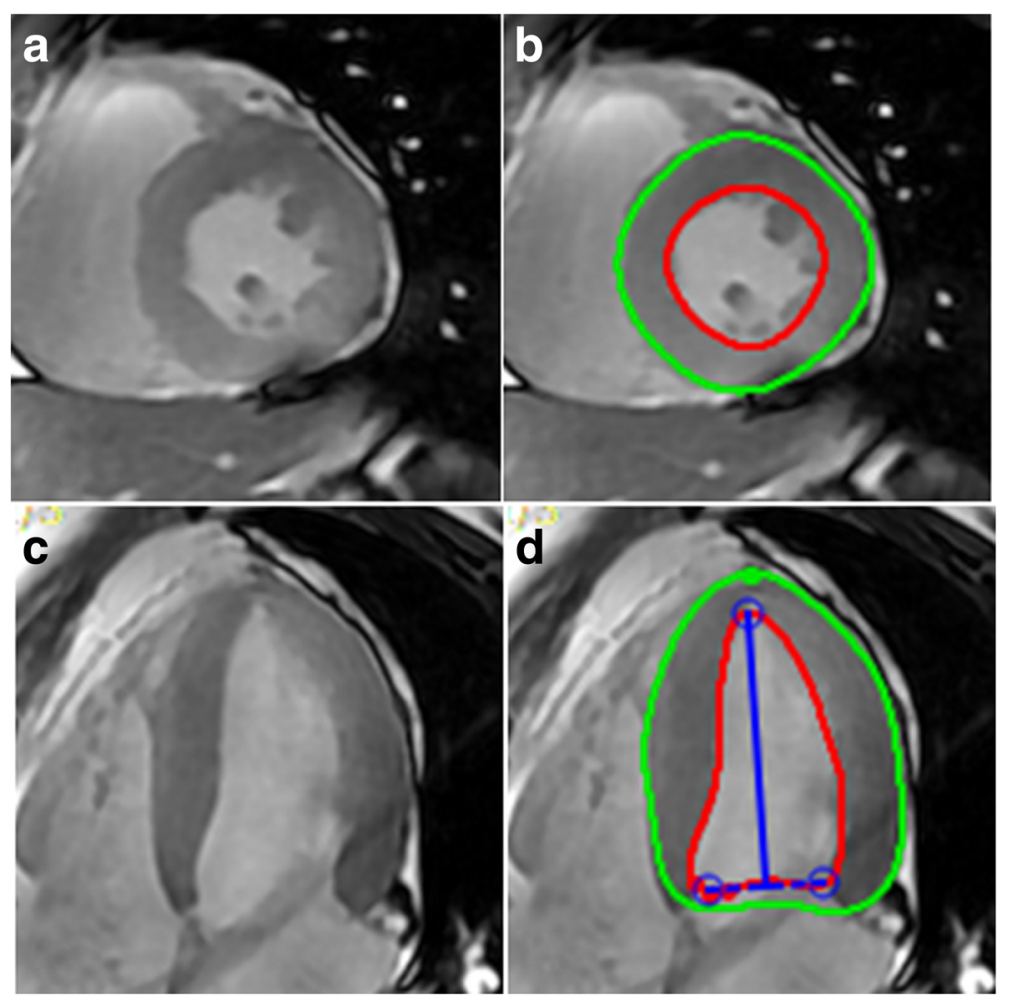

Fig. 1 Contours on cine images for measuring regional myocardial motion indices. a Short-axis view of LV without contours. b Short-axis view of LV with contours. c Four-chamber view of LV without contours. d Four-chamber view of LV with contours 
with the same workflow to test inter-observer agreement of GCA grading and myocardial motion measurements.

\section{Statistics processing}

Data are presented as the mean \pm one standard deviation (SD). GCA scores, LVEF, and regional myocardial motion indices (16 data points on short-axis views and 6 data points on four-chamber views) were compared between two participant groups using the t-test. Pearson's correlation coefficient $(r)$ was applied to test correlations between GCA scores and cardiac motions indices. Bland-Altman plots were used to present intra- and inter-observer variations for measuring regional myocardial motion indices. Inter-observer agreement of GCA grading between two reviewers were evaluated using intra-class correlation coefficient (ICC). Statistical analysis was performed using SPSS software (Version 22.0, IBM, USA). A $p$ value $<0.05$ was considered to demonstrate statistical significance.

\section{Results}

All 22 participants underwent cardiac and head MRI successfully. The image quality of all the participants was eligible for quantitative study. No significant differences in cardiovascular risks were found between the two participant groups (Table 1). Head MRI showed that MCI patients seemed to have a higher average GCA score than healthy controls $(1.18 \pm 0.4$ vs. $0.82 \pm 0.4, p=0.048)$. However, there were no significant differences in LVEF between MCI patients and controls $(52 \% \pm 11 \%$ vs. $55 \% \pm 10 \%, p=0.277)$. Figure 1 shows the contours on the myocardial borders of the LV.

Compared to healthy controls, MCI patients had a lower peak radial strain $(29.1 \% \pm 24.1 \%$ vs. $46.4 \% \pm 43$. $4 \%, p<0.001)$, lower peak diastolic radial strain rate $(3.2$ $\pm 2.4 \mathrm{~s}^{-1}$ vs. $\left.6.0 \pm 3.0 \mathrm{~s}^{-1}, p<0.001\right)$, lower peak diastolic circumferential strain rate $\left(2.5 \pm 2.1 \mathrm{~s}^{-1}\right.$ vs. $3.2 \pm 2.1 \mathrm{~s}^{-1}$, $p=0.002)$, lower peak systolic radial displacement (4.2 \pm $2.2 \mathrm{~mm}$ vs. $5.2 \pm 3.3 \mathrm{~mm}, p=0.002)$, lower peak diastolic radial velocity $(31 \pm 18 \mathrm{~mm} / \mathrm{s}$ vs. $45 \pm 33 \mathrm{~mm} / \mathrm{s}, p<0.001)$, and lower peak diastolic circumferential velocity (178 \pm 124 degree/s vs. $217 \pm 131$ degree/s, $p=0.005)$. See Fig. 2 . However, no significant differences in regional long-axis myocardial motion indices between the two participant groups were found.

There were no significant linear correlations between GCA scores and regional myocardial motion indices in 22 participants. Figure $3(\mathrm{a}-\mathrm{h})$ shows two typical cases (an MCI patient and a healthy control) with different GCA scores and regional myocardial motion patterns through a single cardiac cycle.

Good inter-observer agreement between the two reviewers for GCA grading in all 22 cases was found $(\mathrm{ICC}=0.807)$. Bland-Altman plots showed small intra-
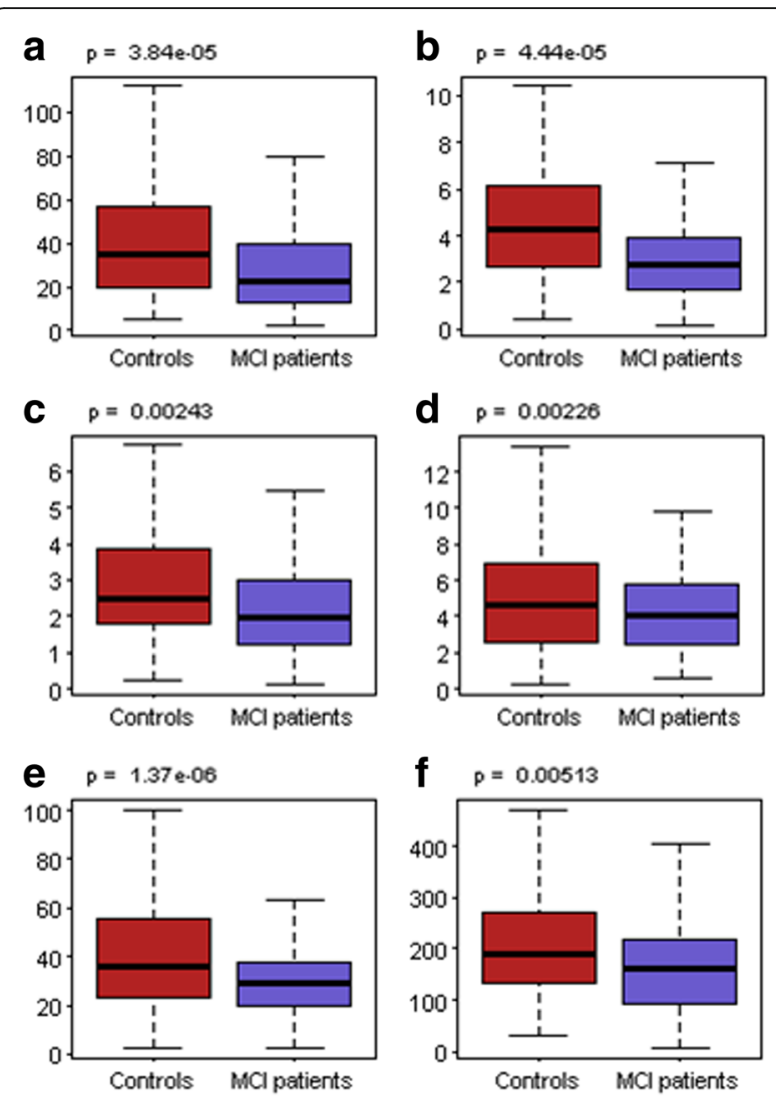

Fig. 2 Differences in regional myocardial motion indices between $\mathrm{MCl}$ patients and healthy controls $(p<0.05)$. a Peak radial strain (\%). b Peak diastolic radial strain rate $\left(s^{-1}\right)$. c Peak diastolic circumferential strain rate $\left(\mathrm{s}^{-1}\right)$. d Peak radial displacement $(\mathrm{mm})$. e Peak diastolic radial velocity $(\mathrm{mm} / \mathrm{s})$. f Peak diastolic circumferential velocity(degree/s)

and inter-observer variations in measuring peak strain at the radial, circumferential and long-axis directions; see Additional file 1.

\section{Discussion}

In the present study, we quantitatively described MRIderived regional myocardial motion patterns from multiple aspects in MCI patients. Compared to sex- and age-matched healthy controls, MCI patients had lower peak regional myocardial strain, strain rate, and velocity in certain directions or time phases. These findings suggest that regional myocardial motion indices, as early indicators of cardiovascular health, are associated with cognitive decline.

Although cognitive decline can be the result of various brain disorders, including tumors, strokes, trauma, infections, neurotoxicity and inherited genetic factors, $\mathrm{AD}$ accounts for 60 to $80 \%$ cognitive impairments in older adults [10]. Almost all AD cases would experience MCI. Some MCI patients would then progress to the advanced stage of AD: AD related dementia (ADRD). Unfortunately, there are no currently effective medications or 

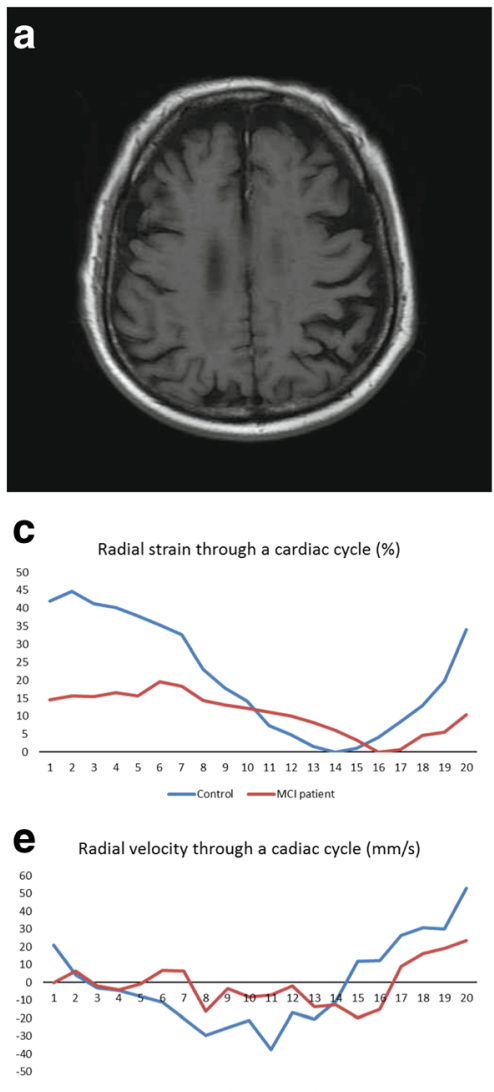

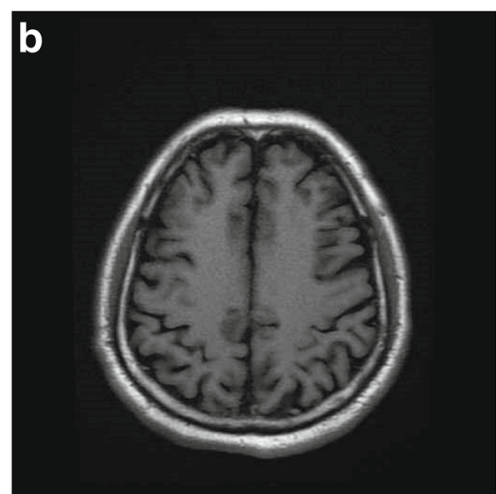

d
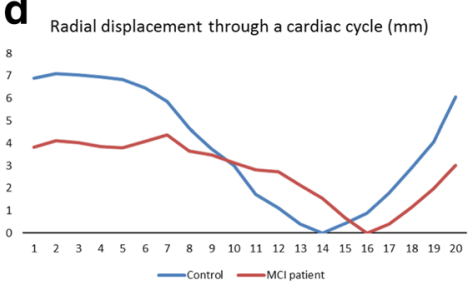

f

Circumferential strain through a cardiac cycle (\%)

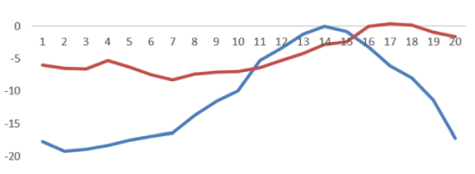

Fig. 3 Comparisons of an MCl patient and a healthy control in manifestations of head MRI and regional myocardial motion patterns. a Participant X, a 69 years old female with MCI. Head MRI (T1WI) shows serious brain atrophy. GCA score is 2. b Participant Y, a 69 years old female control. Head MRI (T1WI) shows mild brain atrophy. GCA score is 1. c Time-resolved curves show that participant $X$ has a lower radial strain than participant $Y$. $\mathbf{d}$ Time-resolved curves show that participant $X$ has a lower peak displacement than participant $Y$. e Time-resolved curves show that participant $X$ has a lower peak velocity (in systole and diastole) than participant Y. $\mathbf{f}$ Time-resolved curves show that participant $X$ has a lower circumferential strain (in systole and diastole) than participant Y

devices that can be used to prevent or treat AD. On the other hand, CVD is also an age-related condition. The existence of CVDs have been closely linked to the development of MCI and dementia [11]. Heart failure, the end stage of heart damage, has been related to cognitive impairments in patients with CVDs [12]. In a cohort study conducted by Robert et al., a total of 2719 participants were followed for 15 months and evaluated for cognitive function using the Clinical Dementia Rating scale. The authors found that CVDs resulted in an increased risk of MCI (risk ratio, 1.77 [95\% confidence interval, 1.16-2.72]) [13]. Rosano et al. demonstrated that the coronary calcium score, a traditional predictor of coronary heart disease (CHD), was related to the incidence of dementia [14]. In addition, Jefferson et al. found that cardiac index was related to information processing speed $(p=0.02)$, brain volume $(p=0.03)$ and lateral ventricular volume $(p=0.048)$ [15]. However, positive correlations between subclinical CVDs and $\mathrm{AD}$ are still not consistent. A double-blind, placebo-controlled trial, the Hypertension in the very
Elderly Trial Cognitive Function Assessment (HYVETCOG) study, reported that anti-hypertension (HTN) treatment with indapamide $(1.5 \mathrm{mg} / \mathrm{d})$ in the elderly $(>80$ years old) did not statistically reduce the incidence of dementia [16]. McGuinness et al. conducted a meta-analysis based on three randomized, double-blind, placebo-controlled trials for the assessment of anti-HTN therapies. However, the authors were unable to confirm that BP decreases in HTN patients could lower the incidence of cognitive impairment or AD [17]. Different interpretations of these conflicting results generated discussion regarding whether " $a$ healthy heart will result in a better brain". Hence, there is no conclusive evidence on whether preventing or treating CVDs benefits $\mathrm{AD}$ management.

Recent studies have shown that CVDs might have a long-standing effect on the brain and therefore recommended early cardiovascular treatment/prevention to benefit the brain [1]. However, current cardiovascular indices presenting global cardiac function, are insufficient for determining $\mathrm{AD}$ risk. Therefore, accurate description 
of regional myocardial motion patterns has high potential to provide critical information for assessing subclinical CVDs and their potential effects on the development of $\mathrm{AD}$. Impaired regional myocardial motion patterns can be observed without a decrease in LVEF in asymptomatic subjects with prominent cardiovascular risk conditions [18-22]. Kresge et al. found that the global longitudinal strain (GLS), a cardiac motion index reflecting the shortening of the entire LV during a cardiac cycle is related to potential cognitive decline in older participants with normal LVEF [23]. In the present study, we further demonstrated that regional myocardial motions indices from the $L V$ were different between $M C I$ patients and healthy controls while there was no significant difference in LVEF. More interestingly, differences in motion indices accompanied divergences in GCA scores in the two participant groups despite there being no direct linear correlations between them. The connections among cardiac MRI, GCA scores and MCI suggest inherent correlations between the existence of subclinical CVDs and neurodegenerative changes. Although further investigations are needed to determine the mechanisms underlying our observations, these cardiac imaging biomarkers have the potential to inform the development of a cost-efficient prevention/treatment strategy simultaneously targeting $\mathrm{CVD}$ and $\mathrm{AD}$, the two prominent threats to aging populations.

The present pilot study had limitations. First, we had a small sample size due to strict enrollment criteria for MCI patients. Therefore, we could only balance prominent cardiovascular risks, such as diabetes mellitus (DM), HTN, and body mass index (BMI), in data analysis. For the same reason, we were unable to distinguish subtypes of MCI (amnestic and nonamnestic) in the present study. Second, according to current cardiac MRI techniques, which might require frequent breath holding during image acquisition, it was difficult to perform cardiac MRI on patients with dementia. As a result, we had to intensively exclude dementia patients. Third, we were unable to acquire regional myocardial motion patterns from the right ventricle (RV) because the thin wall of RV substantially affects the contour drawing and therefore affect the accuracy of motion measurements. Fourth, the aim of the present study was to evaluate the clinical value of regional myocardial motion indices. Therefore, we only ran regular T1WI and T2WI on the participants to obtain GCA despite there being other MRI methods that can represent brain health from other aspects. Fifth, we were unable to determine a causal correlation between changes of regional myocardial motion and cognitive decline. A large-scale, longitudinal study has been designed to further assess the clinical value of cardiovascular imaging biomarkers in the management of $\mathrm{AD}$.

\section{Conclusion}

MRI-derived regional myocardial strain, strain rate and velocity were found to be different between MCI patients and healthy controls. Regional myocardial motion indices have the potential to become novel quantitative imaging biomarkers for representing the risk of neurodegenerative disorders, such as AD.

\section{Additional file}

Additional file 1: Contain Bland-Altman plots for variations in measured regional myocardial motion indices. (DOCX $349 \mathrm{~kb}$ )

\section{Abbreviations}

AD : Alzheimer's disease; CVD : Cardiovascular disease; FOV: Field of view; FSE: Fast spin echo; GCA : Global cortical atrophy; LVEF: Left ventricular ejection fraction; $\mathrm{MCl}$ : Mild cognitive impairment; $\mathrm{MRI}$ : Magnetic resonance imaging; QC: Quality control; ROI: Region of interest

\section{Funding}

This work is supported by the National Natural Science Foundation of China. (81671654, 81401385, and 81571636), Shandong Province Natural. Science Foundation (ZR2014HQ011) and China National Scholarship Fund. (201709370009). KL is supported by a grant from the National Institute of Health (K01HL121162).

\section{Availability of data and materials}

The imaging and clinical information that derived the findings of the present study came from Picture archiving and communication (PACS) system of

Yuhuangding Hospital and were used under license. The clinical information of patients is not publicly available. De-identified data are available from the corresponding author and the first author on reasonable request for any researchers based on a standard agreement of data usage approved by the ethics review committee of Yuhuangding Hospital.

\section{Authors' contributions}

Study design and integrity: HM, JY and KL. Data collection and analysis: JL, HX, FW, XX, WB. Manuscript editing: All authors. All authors read and approved the final manuscript.

Ethics approval and consent to participate

This study was approved by local ethical review committee of Yuhuangding Hospital. All participants signed inform consents before the study.

\section{Competing interests}

The authors declare that they have no competing interests.

\section{Publisher's Note}

Springer Nature remains neutral with regard to jurisdictional claims in published maps and institutional affiliations.

\section{Author details}

${ }^{1}$ Department of Radiology, Yuhuangding Hospital, Qingdao University School of Medicine, 20 E Yuhuangding Rd, Yantai 264000, Shandong, China. 2Department of Radiology, Northwestern University Feinberg School of Medicine, 737 N Michigan Ave, Ste 1600, Chicago, IL 60611, USA.

Received: 18 January 2018 Accepted: 26 April 2018

Published online: 02 May 2018

\section{References}

1. Schievink SHJ, van Boxtel MPJ, Deckers K, van Oostenbrugge RJ, Verhey FRJ, Kohler S. Cognitive changes in prevalent and incident cardiovascular disease: a 12-year follow-up in the Maastricht Aging Study (MAAS). Eur Heart J. 2017. [Epub ahead of print]. 
2. Bancks MP, Allen NB, Dubey P, Launer LI, Lloyd-Jones DM, Reis JP, Sidney S, Yano Y, Schreiner PJ. Cardiovascular health in young adulthood and structural brain MRI in midlife: the CARDIA study. Neurology. 2017;89:680.

3. Bancks MP, Alonso A, Gottesman RF, Mosley TH, Selvin E, Pankow JS. Brain function and structure and risk for incident diabetes: the atherosclerosis risk in communities study. Alzheimers Dement. 2017;13:1345.

4. Santos CY, Snyder PJ, Wu WC, Zhang M, Echeverria A, Alber J. Pathophysiologic relationship between Alzheimer's disease, cerebrovascular disease, and cardiovascular risk: a review and synthesis. Alzheimers Dement (Amst). 2017;7:69-87.

5. Jefferson AL, Beiser AS, Himali JJ, Seshadri S, O'Donnell CJ, Manning WJ, Wolf PA, Au R, Benjamin EJ. Low cardiac index is associated with incident dementia and Alzheimer disease: the Framingham heart study. Circulation. 2015;131(15):1333-9.

6. Jefferson AL, Himali JJ, Au R, Seshadri S, Decarli C, O'Donnell CJ, Wolf PA, Manning WJ, Beiser AS, Benjamin EJ. Relation of left ventricular ejection fraction to cognitive aging (from the Framingham heart study). Am J Cardiol. 2011;108(9):1346-51.

7. Lin K, Collins JD, Chowdhary V, MarkI M, Carr JC. Heart deformation analysis for automated quantification of cardiac function and regional myocardial motion patterns: a proof of concept study in patients with cardiomyopathy and healthy subjects. Eur J Radiol. 2016;85(10):1811-7.

8. Albert MS, DeKosky ST, Dickson D, Dubois B, Feldman HH, Fox NC, Gamst A, Holtzman DM, Jagust WJ, Petersen RC, et al. The diagnosis of mild cognitive impairment due to Alzheimer's disease: recommendations from the National Institute on Aging-Alzheimer's Association workgroups on diagnostic guidelines for Alzheimer's disease. Alzheimers Dement. 2011;7(3):270-9.

9. Wahlund LO, Westman E, van Westen D, Wallin A, Shams S, Cavallin L, Larsson EM. From the imaging cognitive impairment N: imaging biomarkers of dementia: recommended visual rating scales with teaching cases. Insights Imaging. 2017;8(1):79-90.

10. Alzheimer's A. 2016 Alzheimer's disease facts and figures. Alzheimers Dement. 2016;12(4):459-509.

11. Gorelick PB, Furie KL, ladecola C, Smith EE, Waddy SP, Lloyd-Jones DM, Bae HJ, Bauman MA, Dichgans M, Duncan PW, et al. Defining optimal brain health in adults: a presidential advisory from the American Heart Association/American Stroke Association. Stroke. 2017;48(10):e284-303.

12. Heckman GA, Patterson CJ, Demers C, St Onge J, Turpie ID, McKelvie RS Heart failure and cognitive impairment: challenges and opportunities. Clin Interv Aging. 2007;2(2):209-18.

13. Roberts RO, Geda YE, Knopman DS, Cha RH, Pankratz VS, Boeve BF, Tangalos EG, Ivnik RJ, Mielke MM, Petersen RC. Cardiac disease associated with increased risk of nonamnestic cognitive impairment: stronger effect on women. JAMA Neurol. 2013;70(3):374-82.

14. Rosano C, Naydeck B, Kuller LH, Longstreth WT Jr, Newman AB. Coronary artery calcium: associations with brain magnetic resonance imaging abnormalities and cognitive status. J Am Geriatr Soc. 2005;53(4):609-15.

15. Jefferson AL, Himali JJ, Beiser AS, Au R, Massaro JM, Seshadri S, Gona P, Salton CJ, DeCarli C, O'Donnell CJ, et al. Cardiac index is associated with brain aging: the Framingham heart study. Circulation. 2010;122(7):690-7.

16. Tzourio C, Anderson C, Chapman N, Woodward M, Neal B, MacMahon S, Chalmers J, Group PC. Effects of blood pressure lowering with perindopril and indapamide therapy on dementia and cognitive decline in patients with cerebrovascular disease. Arch Intern Med. 2003;163(9):1069-75.

17. McGuinness B, Todd S, Passmore P, Bullock R. The effects of blood pressure lowering on development of cognitive impairment and dementia in patients without apparent prior cerebrovascular disease. Cochrane Database Syst Rev. 2006;2:CD004034.

18. Ernande L, Thibault H, Bergerot C, Moulin P, Wen H, Derumeaux G, Croisille P. Systolic myocardial dysfunction in patients with type 2 diabetes mellitus: identification at MR imaging with cine displacement encoding with stimulated echoes. Radiology. 2012;265(2):402-9.

19. Rider OJ, Ajufo E, Ali MK, Petersen SE, Nethononda R, Francis JM, Neubauer S. Myocardial tissue phase mapping reveals impaired myocardial tissue velocities in obesity. Int J Cardiovasc Imaging. 2015;31(2):339-47.

20. Barbero U, Destefanis P, Pozzi R, Longo F, Piga A. Exercise stress echocardiography with tissue Doppler imaging (TDI) detects early systolic dysfunction in Beta-thalassemia major patients without cardiac Iron overload. Mediterr J Hematol Infect Dis. 2012;4(1):e2012037.

21. Barbero U, Longo F, Destefanis P, Gaglioti CM, Pozzi R, Piga A. Worsening of myocardial performance index in beta-thalassemia patients despite permanently normal iron load at MRI: a simple and cheap index reflecting cardiovascular involvement? IJC Metab Endocr. 2016;13:4.

22. Russo C, Jin Z, Homma S, Elkind MS, Rundek T, Yoshita M, DeCarli C, Wright CB, Sacco RL, Di Tullio MR. Subclinical left ventricular dysfunction and silent cerebrovascular disease: the cardiovascular abnormalities and brain lesions (CABL) study. Circulation. 2013;128(10):1105-11.

23. Kresge HA, Khan OA, Wagener MA, et al. Subclinical Compromise in Cardiac Strain Relates to Lower Cognitive Performances in Older Adults. J Am Heart Assoc. 2018;7(4).

\section{Ready to submit your research? Choose BMC and benefit from:}

- fast, convenient online submission

- thorough peer review by experienced researchers in your field

- rapid publication on acceptance

- support for research data, including large and complex data types

- gold Open Access which fosters wider collaboration and increased citations

- maximum visibility for your research: over $100 \mathrm{M}$ website views per year

At BMC, research is always in progress.

Learn more biomedcentral.com/submissions 